\title{
Geriatric Patients Are at a High Risk of Hypokalemia Associated with Yokukansan Preparation: A Retrospective Cohort Study
}

\author{
Toshinori Hirai, ${ }^{*, a}$ Ryosuke Yamaga, ${ }^{a}$ Motoki Kei, ${ }^{a}$ Keiko Hosohata, ${ }^{b}$ and Toshimasa Itoh ${ }^{a}$ \\ ${ }^{a}$ Department of Pharmacy, Tokyo Women's Medical University Medical Center East; 2-1-10 Nishiogu, Arakawa- \\ ku, Tokyo 116-0011, Japan: and ${ }^{b}$ Education and Research Center for Clinical Pharmacy, Osaka University of \\ Pharmaceutical Sciences; 4-20-1 Nasahara, Takatsuki, Osaka 569-1094, Japan. \\ Received June 6, 2020; accepted August 15, 2020
}

\begin{abstract}
Although hypokalemia is an adverse effect of Yokukansan preparation, especially in geriatric patients, its association with age is unclear. We investigated whether age is a risk factor for hypokalemia. This singlecenter retrospective cohort study, conducted at Tokyo Women's Medical University, Medical Center East between June 2015 and May 2019, included patients who received the Yokukansan preparation. The primary outcome was hypokalemia (serum potassium level: $<3.0 \mathrm{mEq} / \mathrm{L}$ ). A multivariate Cox proportional hazard model was used to determine risk factors, hazard ratio (HR) and $95 \%$ confidence interval $(95 \% \mathrm{CI})$. The cut-off age was also examined. Of 665 patients (median age: 78 years; interquartile range: $68-84$ years), 55 (8.3\%) developed hypokalemia associated with Yokukansan preparation. Risk factors for hypokalemia were age (HR: 1.013, 95\% CI: 1.006-1.021, $p<0.001$ ), dementia (HR: $0.500,95 \%$ CI: $0.357-0.682, p<0.001$ ), serum albumin level (HR: $0.754,95 \%$ CI: $0.669-0.850, p<0.001$ ), and daily Yokukansan preparation dose $\geq 7.5 \mathrm{~g}$ (HR: $1.446,95 \%$ CI: $1.144-1.850, p=0.002$ ). The cut-off ages were $>75$ and $>80$ years but not 65 years and $>70$ years. Clinicians should assess risk factors and monitor serum potassium levels to avoid hypokalemia associated with the Yokukansan preparation.
\end{abstract}

Key words geriatric; hypokalemia; pseudoaldosteronism; Yokukansan; Yokukansankachimpihange

\section{INTRODUCTION}

Yokukansan (Tsumura Co., Tokyo, Japan) is a major Kampo medicine comprising seven crude components (Atractylodes Lancea Rhizome, Poria Sclerotium, Cnidium Rhizome, Uncaria Hook, Angelicae radix, Bupleuri radix, and Glycyrrhiza uralensis). In addition, Yokukansankachimpihange (Tsumura Co., Tokyo, Japan) contains Yokukansan, Citrus unshiu peel, and Pinellia tuber. Several clinical trials have demonstrated that the Yokukansan preparation (comprising Yokukansan and Yokukansankachimpihange) regulates the extracellular level of glutamate and aspartate, and thus alleviates postoperative delirium, behavioral and psychological symptoms of dementia, and symptoms of schizophrenia. ${ }^{1-4)}$

One of the adverse effects of the Yokukansan preparation is hypokalemia, which is attributed to pseudoaldosteronism induced by glycyrrhetinic acid found in Glycyrrhiza (licorice) species. ${ }^{5)}$ Both Yokukansan and Yokukansankachimpihange contain an equivalent amount of Glycyrrhiza species. Several clinical studies and case reports have shown that the Yokukansan preparation could be a cause of hypokalemia and exert harmful effects such as fatigue, arrhythmia, and drowsiness. $^{6-8)}$ Furthermore, hypokalemia associated with the Yokukansan preparation is often observed in geriatric patients. ${ }^{7,8)}$ Especially, geriatric patients with hypokalemia have a low urinary potassium excretion capacity and they have a poor prognosis. ${ }^{9-11)}$ In fact, pseudoaldosteronism is more commonly associated with Yokukansan than other Kampo medicines regardless of relatively low glycyrrhizin content. ${ }^{12)}$ Thus, whether age is a risk factor for hypokalemia associated with the Yokukansan preparation remains to be elucidated.

Hence, we hypothesized that age is involved in the in- cidence of hypokalemia associated with the Yokukansan preparation. In this study, we aimed to test this hypothesis and identify risk factors for hypokalemia associated with the Yokukansan preparation.

\section{MATERIALS AND METHODS}

Study Design and Patients A single-center retrospective cohort study was performed at Tokyo Women's Medical University, Medical Center East (450-bed university hospital in Tokyo, Japan). Patients who started the treatment with the Yokukansan preparation (Yokukansan and Yokukansankachimpihange) between June 2015 and May 2019 were included. Patients were excluded if they met exclusion criteria: 1) patients had no data on serum potassium levels, 2) patients had hypokalemia (serum potassium level $<3.0 \mathrm{mEq} / \mathrm{L}$ ) when the Yokukansan preparation was initiated, or 3) patients were $<20$ years of age. The study design was approved by the Institutional Review Board at Tokyo Women's Medical University (\#5199) and was performed in accordance with the 1964 Helsinki Declaration and its later amendments.

Data Collection We obtained clinical data of patients using the electronic medical records. The clinical data of the study patients were as follows: demographic characteristics (sex, age, height, body weight, and body mass index), main diagnosis (delirium, dementia, and other psychological illness), laboratory data (serum albumin, blood urea nitrogen [BUN], serum creatinine $[\mathrm{Cr}]$, serum sodium, serum potassium, and serum chloride), vital signs (systolic blood pressure, diastolic blood pressure, and heart rate), details of the Yokukansan preparation (dose and follow-up period), and medications of interest (benzodiazepine, ramelteon, suvorexant, cholinester- 
ase inhibitors [donepezil, rivastigmine, and galantamine], $\mathrm{N}$ methyl-D-aspartate antagonist [memantine], typical or atypical antipsychotics, oral corticosteroids, loop diuretics, thiazide diuretics, potassium-sparing diuretics, angiotensin-converting enzyme inhibitors, and angiotensin II receptor blockers). We calculated BUN/Cr based on the ratio of BUN to serum $\mathrm{Cr}$ because it is an indicator of dehydration. In addition, estimated glomerular filtration rate (eGFR) was determined using the prediction equation including sex, age, and serum $\mathrm{Cr}$ level. ${ }^{13)}$ Because we could not obtain blood gas data to assess the presence of acid-base balance disturbance, we calculated the difference between serum sodium and chloride as an alternative marker. $^{14)}$

Outcome The primary outcome was defined as hypokalemia associated with the Yokukansan preparation (serum potassium level: $<3.0 \mathrm{mEq} / \mathrm{L}$ ). The patients were followed up from the initiation of treatment until the date of discontinuation of the Yokukansan preparation or the incidence of hypokalemia.

Statistical Analysis The sample size was estimated as follows. A previous report demonstrated that $24.2 \%$ of patients who received the Yokukansan preparation developed hypokalemia. ${ }^{15)}$ We assumed that $15 \%$ of non-geriatric patients experienced hypokalemia associated with the Yokukansan preparation. Thus, at least 650 patients should be included to have $80 \%$ power in detecting the incidence of hypokalemia associated with the Yokukansan preparation with a two-sided alpha value of 0.05 .

Normally distributed continuous data are expressed as the mean \pm standard deviation and compared using the Student's $t$-test, whereas non-normally distributed data were expressed as median and interquartile range (IQR) and compared using the Mann-Whitney's $U$-test. Categorical data are expressed as numbers and percentages and compared using the chi-square test.

Time-to-event outcomes (hypokalemia associated with the Yokukansan preparation) were analyzed based on KaplanMeier curves, and the log-rank test was used to examine the cumulative incidence of hypokalemia associated with the Yokukansan preparation and its statistical significance. Kaplan-Meier curves were separated by age. The cut-off values of age were defined as 70, 75, and 80 years.

We used a multivariate Cox proportional hazard model to identify the risk factors of hypokalemia associated with the Yokukansan preparation and to calculate the hazard ratio
(HR) and 95\% confidence interval (95\% CI). Hypokalemia associated with the Yokukansan preparation was defined as the dependent variable. The following data were defined as the independent variables: demographic characteristics, main diagnosis, laboratory data, vital signs, dose of the Yokukansan preparation, and medications of interest. The cut-off value for eGFR was $<60 \mathrm{~mL} / \mathrm{min} / 1.73 \mathrm{~m}^{2}$ because this value is used to define chronic kidney disease based on the Chronic Kidney Disease Epidemiology Collaboration equation. ${ }^{16)}$ The dose of the Yokukansan preparation was regarded as a categorical variable and two groups were defined based on the daily dose $(<7.5 \mathrm{~g}$ or $\geq 7.5 \mathrm{~g})$. Medications of interest were categorized as follows: central nervous system medications (benzodiazepine, ramelteon, suvorexant, cholinesterase inhibitors [donepezil, rivastigmine, and galantamine], $N$-methyl-D-aspartate antagonist [memantine] and typical or atypical antipsychotics), potassium-lowering medications (oral corticosteroids, loop diuretics, and thiazide diuretics), and potassium-elevating medications (potassium-sparing diuretics, angiotensin-converting enzyme inhibitors, and angiotensin II receptor blockers).

We screened potential independent variables with $p$-values $<0.10$ by using a univariate Cox proportional hazard model. When there was multicollinearity between any independent variables, we selected one of them with respect to the clinical aspect. We used the stepwise forward selection method to determine the final model based on the Akaike information criterion. The interactions between age and the other independent variables were examined.

We performed a searching analysis to obtain useful data for the risk assessment of hypokalemia associated with the Yokukansan preparation. A multivariate Cox proportional hazard analysis was repeated by substituting age with a categorical variable for a continuous variable in the same manner. The cut-off values of age were defined as 65, 70, 75, and 80 years, because inappropriate medication use was often observed in the $>75$-year-old and $>80$ year-old age groups. ${ }^{17,18)}$

Statistical analyses were performed using $\mathrm{JMP}^{\circledR}$ pro 14 (SAS Institute Inc., Cary, NC, U.S.A.). A $p$-value $<0.05$ was considered statistically significant unless otherwise mentioned.

\section{RESULTS}

Study Patients The flow chart of patient selection is depicted in Fig. 1. There were 732 patients who started the

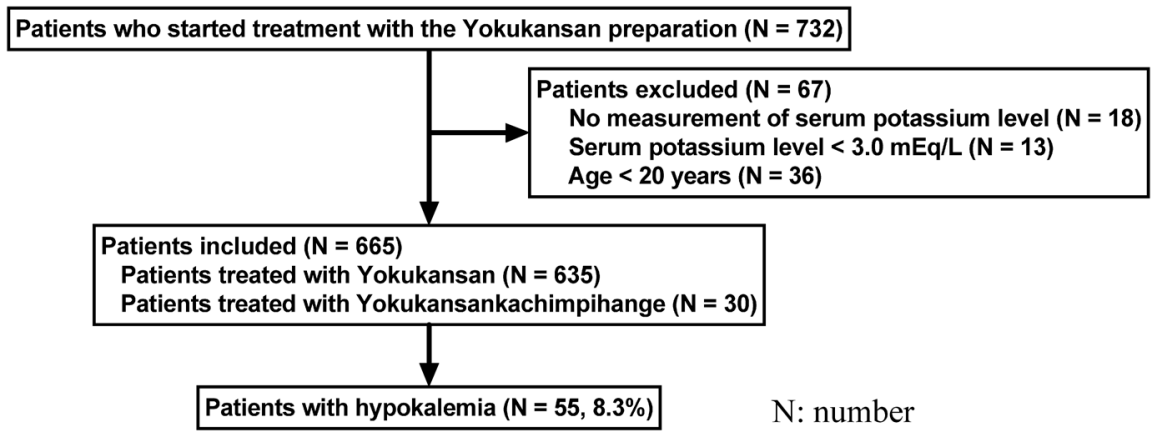

Fig. 1. Flow Chart of Patient Selection

There were 732 patients who underwent the treatment with the Yokukansan preparation at Tokyo Women's Medical University, Medical Center East between June 2015 and May 2019. We excluded 67 patients because serum potassium measurement data were not available for 18 patients, 13 patients had hypokalemia defined as serum potassium level $<3.0 \mathrm{mEq} / \mathrm{L}$, and the remaining 36 patients were $<20$ years of age. The remaining 665 patients were included in this study. Among those included, 635 $(95.5 \%)$ started Yokukansan treatment and the remaining $30(4.5 \%)$ started Yokukansankachimpihange treatment. Hypokalemia (serum potassium levels $<3.0 \mathrm{mEq} / \mathrm{L})$ was observed in $55(8.3 \%)$ patients. 
Yokukansan preparation treatment at our institution between June 2015 and May 2019. We excluded 67 patients because serum potassium levels were not measured for 18 patients, 13 patients have serum potassium level $<3.0 \mathrm{mEq} / \mathrm{L}$ at the treatment initiation, and 36 patients were $<20$ years of age. The remaining 665 patients were included in this study. The clini-

Table 1. Clinical Data of the Study Patients at the Initiation of the Yokukansan Preparation Treatment

\begin{tabular}{|c|c|c|c|}
\hline Variables & Hypokalemia, $N=55$ & No hypokalemia, $N=610$ & $p$-value \\
\hline \multicolumn{4}{|l|}{ Demographic characteristics } \\
\hline Male, $N(\%)$ & $31(56.4)$ & $335(54.9)$ & 0.84 \\
\hline Age, (years) & $79[71-84]$ & $78[68-84]$ & 0.10 \\
\hline Age $>65$ years, $N(\%)$ & $48(87.3)$ & $484(79.3)$ & 0.16 \\
\hline Age $>70$ years, $N(\%)$ & $42(76.4)$ & $432(70.8)$ & 0.38 \\
\hline Age $>75$ years, $N(\%)$ & $33(60.0)$ & $354(58.0)$ & 0.78 \\
\hline Age $>80$ years, $N(\%)$ & $24(43.6)$ & $241(39.5)$ & 0.55 \\
\hline Height, $(\mathrm{cm})$ & $157.2 \pm 10.1$ & $157.9 \pm 9.8$ & 0.67 \\
\hline Body weight, (kg) & $54.1 \pm 12.4$ & $55.4 \pm 12.7$ & 0.49 \\
\hline Body mass index, $\left(\mathrm{kg} / \mathrm{m}^{2}\right)$ & $21.8 \pm 3.9$ & $22.2 \pm 4.1$ & 0.56 \\
\hline \multicolumn{4}{|l|}{ Main diagnosis } \\
\hline Delirium, $N(\%)$ & $33(60.0)$ & $293(48.0)$ & 0.09 \\
\hline Dementia, $N(\%)$ & $7(12.7)$ & $71(11.6)$ & 0.81 \\
\hline Other psychological illness, $N(\%)$ & $15(27.3)$ & $246(40.3)$ & 0.06 \\
\hline \multicolumn{4}{|l|}{ Laboratory data } \\
\hline Serum albumin, (g/dL) & $2.6 \pm 0.79$ & $3.0 \pm 0.76$ & $<0.01$ \\
\hline $\mathrm{BUN},(\mathrm{mg} / \mathrm{dL})$ & $16.3[12.3-27.3]$ & $16.3[11.8-24.1]$ & 0.91 \\
\hline Serum $\mathrm{Cr},(\mathrm{mg} / \mathrm{dL})$ & $0.78[0.56-1.19]$ & $0.80[0.62-1.17]$ & 0.61 \\
\hline $\mathrm{BUN} / \mathrm{Cr}^{\dagger}$ & $18.0[14.2-33.6]$ & $18.9[13.8-26.2]$ & 0.50 \\
\hline $\mathrm{eGFR}^{\star},\left(\mathrm{mL} / \mathrm{min} / 1.73 \mathrm{~m}^{2}\right)$ & $69.3[35.2-88.9]$ & $62.8[42.4-84.9]$ & 0.57 \\
\hline $\mathrm{eGFR}^{\ddagger},<60 \mathrm{~mL} / \mathrm{min} / 1.73 \mathrm{~m}^{2}, N(\%)$ & $23(41.8)$ & $279(45.7)$ & 0.54 \\
\hline Serum $\mathrm{Na},(\mathrm{mEq} / \mathrm{L})$ & $140.0 \pm 5.4$ & $139.4 \pm 5.1$ & 0.39 \\
\hline Serum $K,(m E q / L)$ & $3.8 \pm 0.5$ & $4.1 \pm 0.5$ & $<0.01$ \\
\hline Serum $\mathrm{Cl},(\mathrm{mEq} / \mathrm{L})$ & $102.2 \pm 4.1$ & $102.9 \pm 4.7$ & 0.41 \\
\hline Serum $\mathrm{Na}-$ serum $\mathrm{Cl}^{\S}(\mathrm{mEq} / \mathrm{L})$ & $36.2 \pm 2.5$ & $35.9 \pm 2.9$ & 0.57 \\
\hline \multicolumn{4}{|l|}{ Vital signs } \\
\hline Systolic blood pressure, $(\mathrm{mmHg})$ & $129 \pm 28$ & $133 \pm 24$ & 0.27 \\
\hline Diastolic blood pressure, (mmHg) & $70 \pm 17$ & $73 \pm 14$ & 0.24 \\
\hline Heart rate, (beats per min) & $84 \pm 21$ & $83 \pm 19$ & 0.78 \\
\hline \multicolumn{4}{|l|}{ Detail of Yokukansan preparation } \\
\hline Type of Yokukansan preparation & & & 0.32 \\
\hline Yokukansan, $N(\%)$ & $54(98.2)$ & $581(95.2)$ & \\
\hline Yokukansankachimpihange, $N(\%)$ & $1(1.8)$ & $29(4.8)$ & \\
\hline Yokukansan preparation dose $\geq 7.5 \mathrm{~g} / \mathrm{d}, N(\%)$ & $45(81.8)$ & $484(79.3)$ & 0.66 \\
\hline Follow-up period, (days) & $38[20-139]$ & $26[12-113]$ & 0.03 \\
\hline \multicolumn{4}{|l|}{ Medications of interest } \\
\hline Central nervous system medications, $N(\%)$ & $45(81.8)$ & $436(71.5)$ & 0.10 \\
\hline Benzodiazepine, $N(\%)$ & $13(23.6)$ & $127(20.8)$ & 0.62 \\
\hline Ramelteon, $N(\%)$ & $21(38.2)$ & $213(34.9)$ & 0.63 \\
\hline Suvorexant, $N(\%)$ & $6(10.9)$ & $44(7.2)$ & 0.32 \\
\hline Cholinesterase inhibitors $\mathbb{\AA}, N(\%)$ & $6(10.9)$ & $57(9.3)$ & 0.70 \\
\hline Memantine, $N(\%)$ & $4(7.3)$ & $32(5.2)$ & 0.52 \\
\hline Typical antipsychotics, $N(\%)$ & $2(3.6)$ & $41(6.7)$ & 0.37 \\
\hline Atypical antipsychotics, $N(\%)$ & $14(25.5)$ & $100(16.4)$ & 0.09 \\
\hline Potassium-lowering medications, $N(\%)$ & $17(30.9)$ & $117(19.2)$ & 0.04 \\
\hline Oral corticosteroids, $N(\%)$ & $4(7.3)$ & $29(4.8)$ & 0.41 \\
\hline Loop diuretics, $N(\%)$ & $14(25.5)$ & $82(13.4)$ & 0.02 \\
\hline Thiazide diuretics, $N(\%)$ & $0(0)$ & $15(2.5)$ & 0.24 \\
\hline Potassium-elevating medications, $N(\%)$ & $25(45.5)$ & $183(30.0)$ & 0.02 \\
\hline Potassium-sparing diuretics, $N(\%)$ & $13(23.6)$ & $47(7.7)$ & $<0.01$ \\
\hline $\mathrm{ACEi}$ or $\mathrm{ARB}, N(\%)$ & $18(32.7)$ & $155(25.4)$ & 0.24 \\
\hline
\end{tabular}

N: number, BUN: blood urea nitrogen, Cr: creatinine, eGFR: estimated glomerular filtration rate, Na: sodium, $\mathrm{K}$ : potassium, Cl: chloride, ACEi: angiotensin-convertingenzyme inhibitor, ARB: angiotensin II receptor blocker Normally distributed continuous data are expressed as mean \pm standard deviation and compared using the Student's $t$ test. Non-normally distributed data are expressed as median and interquartile range and compared using the Mann-Whitney's $U$-test. Categorical data are expressed as numbers and percentages and compared using the chi-square test. $\uparrow:$ BUN/Cr is a ratio of blood urea nitrogen and serum creatinine. $\$$ : eGFR was calculated from the prediction equation based on sex, age, and serum creatinine level. $\S$ : we calculated serum $\mathrm{Na}$-serum $\mathrm{Cl}$ to assess the presence of acid-base balance disturbance: $\mathbf{I}$ : Cholinesterase inhibitors included donepezil, rivastigmine, and galantamine. 

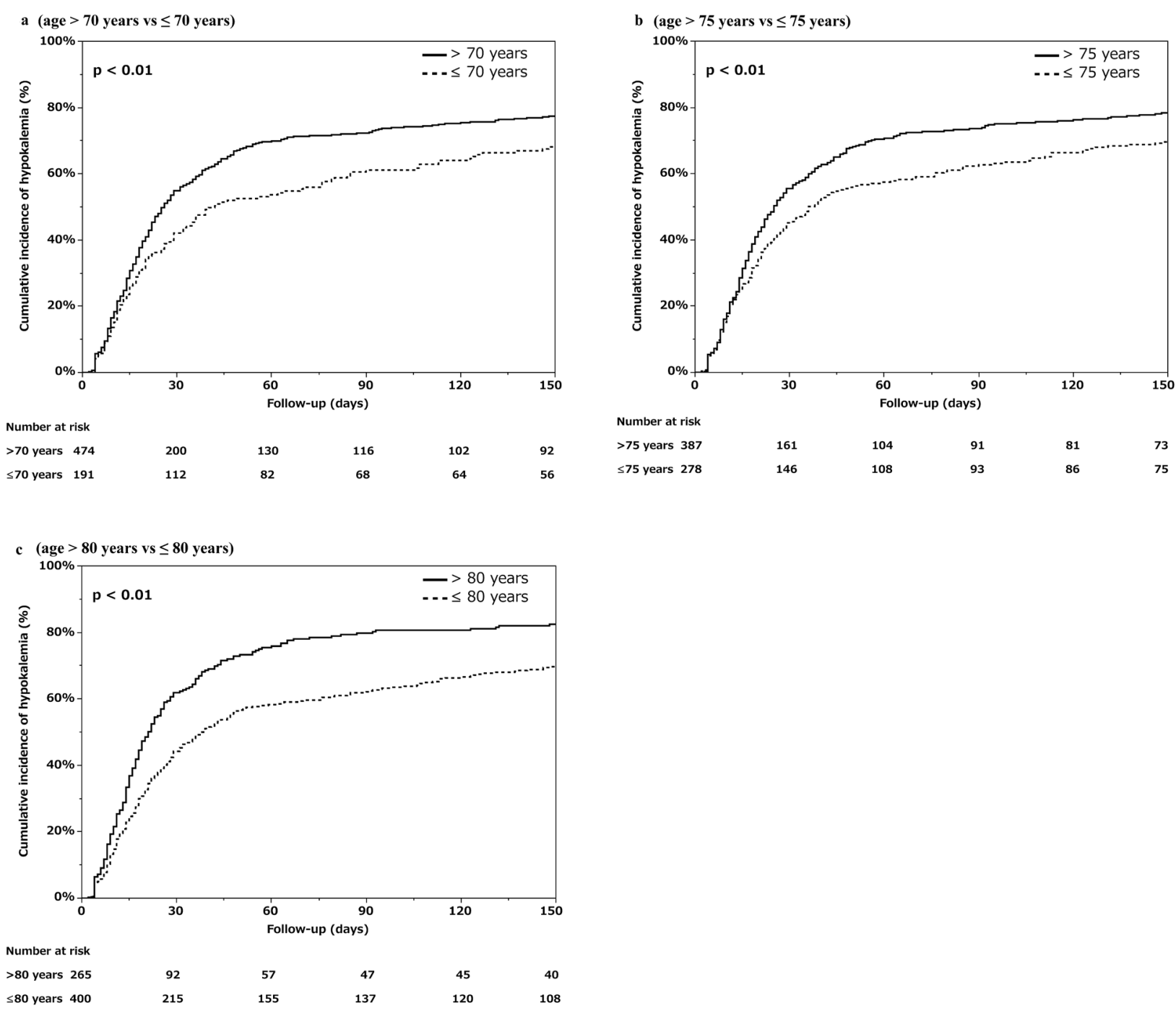

Fig. 2. Kaplan-Meier Curves for Hypokalemia Associated with the Yokukansan Preparation between Different Age Groups

The $X$ - and $Y$-axes represent the follow-up period and the cumulative incidence of hypokalemia associated with the Yokukansan preparation, respectively. Kaplan-Meier curves for the cumulative incidence of hypokalemia associated with the Yokukansan preparation were stratified according to the cut-off values of age as follows: 70 years (Fig. 2a), 75 years (Fig. 2b), and 80 years (Fig. 2c). Continuous lines represent a group of geriatric patients ( $>70$ years, $>75$ years, and $>80$ years). Dotted lines represent a group of non-geriatric patients ( $\leq 70$ years, $\leq 75$ years, and $\leq 80$ years). The number at risk, stratified by age, is shown below the $X$-axis. Comparison of Kaplan-Meier curves were generated using the log-rank test. The cumulative incidence of hypokalemia associated with the Yokukansan preparation was high in all geriatric patients (all groups: $p<0.01$ )

cal data of the study patients are summarized in Table 1. Of the study patients, $366(55.0 \%)$ were male. The median patient age was 78 years (IQR: 68-84). Furthermore, 635 (95.5\%) patients started the Yokukansan treatment and the remaining $30(4.5 \%)$ started the Yokukansankachimpihange treatment. There were $529(79.5 \%)$ patients who started treatment with daily Yokukansan preparation dose of $\geq 7.5 \mathrm{~g}$. The median follow-up period was $27 \mathrm{~d}$ (IQR: 14-113).

Outcome Among the study patients, 55 (8.3\%) developed hypokalemia associated with the Yokukansan preparation during the median follow-up period of $38 \mathrm{~d}$ (IQR: 20-139). The cumulative incidence of hypokalemia associated with the Yokukansan preparation differed according to age groups (Fig. 2). The cumulative incidence of hypokalemia associated with the Yokukansan preparation was higher in geriatric patients than in non-geriatric patients (for all groups: $p<0.01$ ).

Data Analysis The univariate Cox proportional hazard model revealed that male sex, age, delirium, dementia, serum albumin level, BUN/Cr, daily Yokukansan preparation dose
Table 2. Multivariate Cox Proportional Hazard Model for Hypokalemia Associated with the Yokukansan Preparation

\begin{tabular}{lccr}
\hline \hline Independent variables & HR & $95 \% \mathrm{CI}$ & $p$-value \\
\hline Age (per years) & 1.013 & $1.006-1.021$ & $<0.001$ \\
Dementia (present) & 0.500 & $0.357-0.682$ & $<0.001$ \\
Serum albumin (per g/dL) & 0.754 & $0.669-0.850$ & $<0.001$ \\
Yokukansan preparation dose ${ }^{\dagger}$ & 1.446 & $1.144-1.850$ & 0.002 \\
$\quad \geq 7.5 \mathrm{~g} / \mathrm{d}$ (yes) & & & \\
\hline
\end{tabular}

HR: hazard ratio, 95\% CI: 95\% confidence interval. †: Yokukansan and Yokukansankachimpihange.

of $\geq 7.5 \mathrm{~g}$, and potassium-lowering medications were potential independent variables influencing hypokalemia associated with the Yokukansan preparation. No multicollinearity between potential independent variables was confirmed. Based on the stepwise forward selection method, age, dementia, serum albumin level, and daily Yokukansan preparation dose $\geq 7.5 \mathrm{~g}$ were included in the final model of hypokalemia asso- 
Table 3. Multivariate Cox Proportional Hazard Model for Hypokalemia Associated with the Yokukansan Preparation

\begin{tabular}{|c|c|c|c|c|c|c|c|c|c|}
\hline \multirow{2}{*}{ Independent variables } & \multicolumn{3}{|c|}{ Model 1 (age $>70$ years) } & \multicolumn{3}{|c|}{ Model 2 (age $>75$ years) } & \multicolumn{3}{|c|}{ Model 3 (age $>80$ years) } \\
\hline & $\mathrm{HR}$ & $95 \% \mathrm{CI}$ & $p$-value & $\mathrm{HR}$ & $95 \% \mathrm{CI}$ & $p$-value & HR & $95 \% \mathrm{CI}$ & $p$-value \\
\hline \multicolumn{10}{|l|}{ Age group } \\
\hline Age $>70$ years $($ yes $)$ & 1.211 & $0.974-1.515$ & 0.085 & - & - & - & - & - & - \\
\hline Age $>75$ years $($ yes) & - & - & - & 1.251 & $1.036-1.512$ & 0.020 & - & - & - \\
\hline Age $>80$ years $($ yes $)$ & - & - & - & - & - & - & 1.450 & $1.198-1.749$ & $<0.001$ \\
\hline Delirium (present) & 1.225 & $0.981-1.536$ & 0.073 & - & - & - & - & - & - \\
\hline Dementia (present) & 0.594 & $0.416-0.831$ & 0.003 & 0.545 & $0.391-0.741$ & $<0.001$ & 0.547 & $0.393-0.743$ & $<0.001$ \\
\hline Serum albumin (per g/dL) & 0.754 & $0.666-0.855$ & $<0.001$ & 0.722 & $0.643-0.811$ & $<0.001$ & 0.728 & $0.648-0.818$ & $<0.001$ \\
\hline Yokukansan preparation dose ${ }^{\dagger} \geq 7.5 \mathrm{~g} / \mathrm{d}$ (yes) & 1.387 & $1.093-1.781$ & 0.007 & 1.460 & $1.154-1.870$ & 0.001 & 1.477 & $1.168-1.892$ & $<0.001$ \\
\hline
\end{tabular}

HR: hazard ratio, 95\% CI: 95\% confidence interval. †: Yokukansan and Yokukansankachimpihange.

ciated with the Yokukansan preparation. The results obtained using the multivariate Cox proportional hazard model demonstrated that age (HR: 1.013, 95\% CI: 1.006-1.021, $p<0.001$ ), dementia (HR: 0.500, 95\% CI: $0.357-0.682, p<0.001$ ), serum albumin level (HR: 0.754, 95\% CI: 0.669-0.850, $p<0.001$ ), and daily Yokukansan preparation dose $\geq 7.5 \mathrm{~g}$ (HR: 1.446, 95\% CI: $1.144-1.850, p=0.002$ ) significantly influenced the development of hypokalemia associated with the Yokukansan preparation (Table 2). No interaction between age and the other independent variables was observed.

The searching analysis showed that the age category of $>65$ years was not included in the final model for the development of hypokalemia associated with the Yokukansan preparation. However, age $>75$ years and $>80$ years, but not $>70$ years, were independent risk factors for the development of hypokalemia associated with the Yokukansan preparation (Table 3). In addition, dementia, serum albumin level, and daily Yokukansan preparation dose $\geq 7.5 \mathrm{~g}$ were risk factors for the development of hypokalemia associated with the Yokukansan preparation even if age was used as a categorical variable.

\section{DISCUSSION}

The results of the present study demonstrated that old age is significantly associated with the development of hypokalemia associated with the Yokukansan preparation, and this is in line with our hypothesis. In addition, dementia, serum albumin level, and daily Yokukansan preparation dose $\geq 7.5 \mathrm{~g}$ were also clinical predictors for the development of hypokalemia associated with the Yokukansan preparation.

The fact that approximately $40 \%$ of the patients in our study were $>80$ years of age is noteworthy. In previous studies, patients who developed hypokalemia associated with the Yokukansan preparation or licorice-containing herbal medicine were older than 70 years, except for only a few patients. ${ }^{6,7,19)}$ We consider that increased age reflects a high prevalence of hypokalemia associated with the Yokukansan preparation. In general, among geriatric patients, hypokalemia is associated with the length of hospital stay, medical cost, and an unfavorable prognosis. ${ }^{20)}$ It is recommended that serum potassium level should be carefully managed in geriatric patients receiving the Yokukansan preparation treatment.

With regard to the mechanism underlying the relationship between age and hypokalemia associated with the Yokukansan preparation, eGFR was compatible, irrespective of the presence of hypokalemia with the Yokukansan preparation (data not shown). 11 $\beta$-Hydroxysteroid dehydrogenase type 2 activity plays a major role in the development of hypokalemia associated with the Yokukansan preparation. ${ }^{21)}$ Glycyrrhetinic acid inhibits $11 \beta$-hydroxysteroid dehydrogenase type 2 and increases the excretion of potassium. ${ }^{21,22)}$ Henschkowski et al. demonstrated that $11 \beta$-hydroxysteroid dehydrogenase type 2 activity was inversely correlated with age independent of kidney function. ${ }^{23}$ These findings could support the relationship between age and hypokalemia associated with the Yokukansan preparation. Although we could not evaluate $11 \beta$-hydroxysteroid dehydrogenase type 2 activity, age could be a predictor for the development of hypokalemia associated with the Yokukansan preparation. In addition, metabolites of glycyrrhetic acid such as monoglucuronide and sulfate could be a cause of pseudoaldosteronism. ${ }^{24)}$ Further analysis is required to identify the relationship between metabolites and older age.

Dementia was associated with a decrease in the incidence of hypokalemia associated with the Yokukansan preparation. Although the underlying reason is not clear, patients with dementia may react poorly to the Yokukansan preparation because they have poor adherence and abnormal intestinal bacterial flora which is an indispensable factor behind the bioactivation of Glycyrrhetinic acid. ${ }^{25)}$ Furthermore, patients with dementia have a low eGFR, resulting in a low potassium excretion rate and a high serum albumin level (data not shown). This reflects a relatively high potassium retention status compared with that in patients without dementia. Further research is warranted to clarify the mechanism between dementia and Yokukansan preparation.

We also attempted to evaluate the relationship between hypokalemia associated with the Yokukansan preparation and medications that affect serum potassium levels. However, both potassium-lowering medications and potassium-elevating medications did not significantly influence the incidence of hypokalemia associated with the Yokukansan preparation. We assume that the frequency of use of these medications was quite low. Moreover, previous studies have indicated that elderly patients have a large interindividual variation in furosemide bioavailability ${ }^{26)}$ and that hypoalbuminemia reduces diuretic response. ${ }^{27)}$ Thus, the individual differences in concurrent medications might be an important risk factor for hypokalemia.

Many studies have demonstrated that elderly patients with hypokalemia associated with the Yokukansan prepara- 
tion show hypoalbuminemia as well. ${ }^{6,15,28)}$ In this study, we found that patients with hypoalbuminemia were at a higher risk of hypokalemia (Table 2). Glycyrrhetic acid, the active metabolite of glycyrrhizin, is metabolized via several uridine diphosphate-glucuronosyltransferases, ${ }^{29)}$ and in a basic experiment, it was reported that the presence of bovine albumin elevates the capacity of glucuronidation. ${ }^{29)}$ Thus, hypoalbuminemia might alter the exposure to glycyrrhetinic acid and increase the risk of hypokalemia. Although the serum albumin level is a marker for a variety of conditions (e.g. edema, cirrhosis, and kidney disease), it is notable that hypoalbuminemia could induce metabolic alkalosis influencing the homeostatic mechanism of potassium. ${ }^{30)}$ In addition, we consider that the main reason for hypoalbuminemia is an undernutrition condition resulting in hypokalemia. Indeed, geriatric patients with undernutrition often show hypoalbuminemia. ${ }^{31)}$ Furthermore, hypokalemia is associated with undernutrition. ${ }^{32)}$ Hypoalbuminemia comprises accessible information to assess the risk of hypokalemia associated with the Yokukansan preparation in clinical practice.

Daily Yokukansan preparation dose of $\geq 7.5 \mathrm{~g}$ is a risk factor for hypokalemia, as reported previously. ${ }^{15)}$ Daily Yokukansan preparation dose of $7.5 \mathrm{~g}$ contains $1.5 \mathrm{~g}$ of Glycyrrhiza, which corresponds to a relatively small amount of licorice among Kampo medicines. Moreover, daily Yokukansan dose of $7.5 \mathrm{~g}$ has been employed in many clinical trials. ${ }^{33)}$ Thus, the evidence-based use of Yokukansan treatment might expose patients to a risk for hypokalemia, associated with the Yokukansan preparation, in clinical practice. Additional analyses are warranted to assess the association between hypokalemia and a lot of Kampo medicines containing various amounts of Glycyrrhiza species, such as Shakuyakukanzoto and Rikkunshito.

The searching analysis implied that the incidence of hypokalemia associated with the Yokukansan preparation increased with advancing age. In a study in which the mean patient age was 76.7 years, potentially inappropriate Kampo medications were confirmed with approximately $30 \%$ of patients. ${ }^{34)} \mathrm{We}$ could not evaluate the effect of the other age groups (e.g., 50 years) because patients aged $<50$ years were limited in our study. In particular, the need for Yokukansan preparations should be evaluated in elderly patients $>75$ years of age.

These results should be interpreted with caution since there are some limitations to this study. First, this was a singlecenter retrospective observational study. Therefore, we could not avoid bias. Second, there was no data available regarding food intake, the main diagnosis (the type of delirium and severity of dementia), urinary electrolyte analysis, and endocrinological parameters such as renin, aldosterone, and cortisol. Third, we could not assess whether patients had an acid-base disorder induced by the Yokukansan preparation because the blood gas findings were not obtained. Fourth, some laboratory and vital sign data were missing. Finally, the frequency of serum potassium measurements was inconsistent. Thus, the incidence of hypokalemia associated with Yokukansan might be inaccurate.

In summary, geriatric patients are at a high risk of developing hypokalemia associated with the Yokukansan preparation. Additionally, clinicians should pay attention to hypoalbuminemia and high doses of the Yokukansan preparation to detect patients at a high risk of developing hypokalemia associated with the Yokukansan preparation. Close monitoring of serum potassium levels should be considered for patients at a high risk of hypokalemia.

Conflict of Interest The authors declare no conflict of interest.

\section{REFERENCES}

1) Okahara $K$, Ishida $Y$, Hayashi $Y$, Inoue T, Tsuruta $K$, Takeuchi $K$, Yoshimuta H, Kiue K, Ninomiya Y, Kawano J, Yoshida K, Noda S, Tomita S, Fujimoto M, Hosomi J, Mitsuyama Y. Effects of Yokukansan on behavioral and psychological symptoms of dementia in regular treatment for Alzheimer's disease. Prog. Neuropsychopharmacol. Biol. Psychiatry, 34, 532-536 (2010).

2) Miyaoka T, Furuya M, Yasuda H, Hayashida M, Nishida A, Inagaki T, Horiguchi J. Yi-gan san as adjunctive therapy for treatment-resistant schizophrenia: an open-label study. Clin. Neuropharmacol., 32, 6-9 (2009).

3) Wada S, Inoguchi H, Hirayama T, Matsuoka YJ, Uchitomi Y, Ochiai H, Tsukamoto S, Shida D, Kanemitsu Y, Shimizu K. Yokukansan for the treatment of preoperative anxiety and postoperative delirium in colorectal cancer patients: a retrospective study. Jpn. J. Clin. Oncol., 47, 844-848 (2017).

4) Takeda A, Itoh $H$, Tamano $H$, Yuzurihara M, Oku N. Suppressive effect of Yokukansan on excessive release of glutamate and aspartate in the hippocampus of zinc-deficient rats. Nutr. Neurosci., 11, 41-46 (2008).

5) Conn JW, Rovner DR, Cohen EL. Licorice-induced pseudoaldosteronism. Hypertension, hypokalemia, aldosteronopenia, and suppressed plasma renin activity. JAMA, 205, 492-496 (1968).

6) Kamei $\mathrm{S}$, Kaneto $\mathrm{H}$, Irie $\mathrm{S}$, Kinoshita $\mathrm{T}$, Tanabe A, Hirukawa $\mathrm{H}$, Tatsumi F, Shimoda M, Kohara K, Mune T, Kaku K. Pseudoaldosteronism induced by Yokukansan in an elderly Japanese type 2 diabetic patient with Alzheimer's disease. J. Diabetes. Investig., 6, 487-488 (2015).

7) Yamada S, Tokumoto M, Kansui Y, Wakisaka Y, Uchizono Y, Tsuruya K, Ooboshi H. Severe metabolic alkalosis, hypokalemia, and respiratory acidosis induced by the Chinese herbal medicine yokukansan in an elderly patient with muscle weakness and drowsiness. CEN Case Rep., 2, 23-27 (2013).

8) Mizukami K, Asada T, Kinoshita $\mathrm{T}$, et al. A randomized cross-over study of a traditional Japanese medicine (kampo), yokukansan, in the treatment of the behavioural and psychological symptoms of dementia. Int. J. Neuropsychopharmacol., 12, 191-199 (2009).

9) Musso C, Liakopoulos V, De Miguel R, Imperiali N, Algranati L. Transtubular potassium concentration gradient: comparison between healthy old people and chronic renal failure patients. Int. Urol. Nephrol., 38, 387-390 (2006).

10) Jensen HK, Brabrand M, Vinholt PJ, Hallas J, Lassen AT. Hypokalemia in acute medical patients: risk factors and prognosis. Am. $J$. Med., 128, 60-67.e1 (2015).

11) Tazmini K, Nymo SH, Louch WE, Ranhoff AH, Oie E. Electrolyte imbalances in an unselected population in an emergency department: A retrospective cohort study. PLOS ONE, 14, e0215673 (2019)

12) Nose $M$, Tada $M$, Kojima $R$, Nagata $K$, Hisaka $S$, Masada $S$, Homma M, Hakamatsuka T. Comparison of glycyrrhizin content in 25 major kinds of Kampo extracts containing Glycyrrhizae Radix used clinically in Japan. J. Nat. Med., 71, 711-722 (2017).

13) Matsuo S, Imai E, Horio M, Yasuda Y, Tomita K, Nitta K, Yamagata $\mathrm{K}$, Tomino $\mathrm{Y}$, Yokoyama $\mathrm{H}$, Hishida A. Revised equations for estimated GFR from serum creatinine in Japan. Am. J. Kidney Dis., 53, 982-992 (2009).

14) Nagaoka D, Nassar Junior AP, Maciel AT, Taniguchi LU, Noritomi DT, Azevedo LC, Neto LM, Park M. The use of sodium-chloride 
difference and chloride-sodium ratio as strong ion difference surrogates in the evaluation of metabolic acidosis in critically ill patients. J. Crit. Care, 25, 525-531 (2010).

15) Shimada S, Arai T, Tamaoka A, Homma M. Liquorice-induced hypokalaemia in patients treated with Yokukansan preparations: identification of the risk factors in a retrospective cohort study. $B M J$ Open, 7, e014218 (2017).

16) Levey AS, Stevens LA, Schmid CH, Zhang YL, Castro AF 3rd, Feldman HI, Kusek JW, Eggers P, Van Lente F, Greene T, Coresh J. A new equation to estimate glomerular filtration rate. Ann. Intern. Med., 150, 604-612 (2009).

17) Sarwar MR, Dar AR, Mahar SY, Riaz T, Danish U, Iftikhar S. Assessment of prescribing potentially inappropriate medications listed in Beers criteria and its association with the unplanned hospitalization: a cross-sectional study in Lahore, Pakistan. Clin. Interv. Aging, 13, 1485-1495 (2018)

18) Lai $X$, Zhu H, Huo $X$, Li Z. Polypharmacy in the oldest old $(>/=80$ years of age $)$ patients in China: a cross-sectional study. BMC Geriatr., 18, 64 (2018).

19) Kurisu S, Inoue I, Kawagoe T, Ishihara M, Shimatani $Y$, Nakama Y, Maruhashi T, Kagawa E, Dai K, Aokage T, Matsushita J, Ikenaga H. Clinical profile of patients with symptomatic glycyrrhizininduced hypokalemia. J. Am. Geriatr. Soc., 56, 1579-1581 (2008).

20) Bardak S, Turgutalp K, Koyuncu MB, Hari H, Helvaci I, Ovla D, Horoz M, Demir S, Kiykim A. Community-acquired hypokalemia in elderly patients: related factors and clinical outcomes. Int. Urol. Nephrol., 49, 483-489 (2017).

21) Kageyama Y, Suzuki H, Saruta T. Glycyrrhizin induces mineralocorticoid activity through alterations in cortisol metabolism in the human kidney. J. Endocrinol., 135, 147-152 (1992).

22) Kato H, Kanaoka M, Yano S, Kobayashi M. 3-Monoglucuronylglycyrrhetinic acid is a major metabolite that causes licoriceinduced pseudoaldosteronism. J. Clin. Endocrinol. Metab., 80, 1929-1933 (1995).

23) Henschkowski J, Stuck AE, Frey BM, Gillmann G, Dick B, Frey FJ, Mohaupt MG. Age-dependent decrease in 11beta-hydroxysteroid dehydrogenase type 2 (11beta-HSD2) activity in hypertensive patients. Am. J. Hypertens., 21, 644-649 (2008).

24) Morinaga O, Ishiuchi K, Ohkita T, Tian C, Hirasawa A, Mitamura M, Maki Y, Yasujima T, Yuasa H, Makino T. Isolation of a novel glycyrrhizin metabolite as a causal candidate compound for pseudoaldosteronism. Scientific Reports, 8, 15568 (2018).

25) Hattori M, Sakamoto T, Yamagishi T, Sakamoto K, Konishi K, Kobashi K, Namba T. Metabolism of glycyrrhizin by human intestinal flora. II. Isolation and characterization of human intestinal bacteria capable of metabolizing glycyrrhizin and related compounds. Chem. Pharm. Bull., 33, 210-217 (1985).

26) Murray MD, Haag KM, Black PK, Hall SD, Brater DC. Variable furosemide absorption and poor predictability of response in elderly patients. Pharmacotherapy, 17, 98-106 (1997).

27) ter Maaten JM, Valente MA, Damman K, Hillege HL, Navis G, Voors AA. Diuretic response in acute heart failure-pathophysiology, evaluation, and therapy. Nat. Rev. Cardiol., 12, 184-192 (2015).

28) Yasue H, Itoh T, Mizuno Y, Harada E. Severe hypokalemia, rhabdomyolysis, muscle paralysis, and respiratory impairment in a hypertensive patient taking herbal medicines containing licorice. Intern. Med., 46, 575-578 (2007).

29) Lu Y, Zhu J, Chen X, Li N, Fu F, He J, Wang G, Zhang L, Zheng Y, Qiu Z, Yu X, Han D, Wu L. Identification of human UDP-glucuronosyltransferase isoforms responsible for the glucuronidation of glycyrrhetinic acid. Drug Metab. Pharmacokinet., 24, 523-528 (2009).

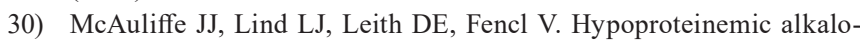
sis. Am. J. Med., 81, 86-90 (1986).

31) Brock F, Bettinelli LA, Dobner T, Stobbe JC, Pomatti G, Telles CT. Prevalence of hypoalbuminemia and nutritional issues in hospitalized elders. Rev. Lat. Am. Enfermagem, 24, e2736 (2016).

32) Wang HH, Hung CC, Hwang DY, Kuo MC, Chiu YW, Chang JM, Tsai JC, Hwang SJ, Seifter JL, Chen HC. Hypokalemia, its contributing factors and renal outcomes in patients with chronic kidney disease. PLOS ONE, 8, e67140 (2013).

33) Okamoto H, Iyo M, Ueda K, Hirasaki Y, Namiki T, Han C. Yokukan-san: a review of the evidence for use of this Kampo herbal formula in dementia and psychiatric conditions. Neuropsychiatr. Dis. Treat., 10, 1727-1742 (2014)

34) Komagamine J, Hagane K. Prevalence of the potentially inappropriate Kampo medications to be used with caution among elderly patients taking any prescribed Kampo medications at a single centre in Japan: a retrospective cross-sectional study. BMC Complement. Altern. Med., 18, 155 (2018). 